\title{
Análisis geoespacial de las elecciones presidenciales en México, 2012
}

Vadimir Hernández-Hernández. Universidad Autónoma de Ciudad Juárez, Ciudad Juárez, México.

RESUMEN | La geografía electoral, en tanto rama de la geografía política, brinda la oportunidad de explicar las preferencias de voto en función de la organización territorial, esto es, de los lugares en que ocurren las elecciones. Los estudios electorales con un enfoque geográfico suponen que las preferencias políticas individuales que se dan un sitio no son independientes entre sí, sino que, por el contrario, están correlacionadas. Este artículo aborda la existencia de una geografía del voto presidencial en las elecciones de 2012 en México. Luego de revisar la literatura y argumentar el uso del análisis exploratorio de datos espaciales, se propone el empleo de índices de autocorrelación espacial para el estudio de dichas elecciones. El esquema propuesto, que da cuenta de la polarización política territorial en México, permite valorar el poder explicativo del espacio geográfico en los procesos electorales.

PALABRAS CLAVE | concentración espacial, transformaciones socioterritoriales, distribución espacial.

ABSTRACT | The importance of electoral geography, as a branch of political geography, stems from the opportunity it provides to explain electoral preferences in terms of territories. Electoral research with a geographic focus assumes that individual political preferences concentrated in a particular location are not unrelated, but are in fact correlated. This article discusses the existence of a geography of the presidential vote in the 2012 elections in Mexico. After reviewing the literature and arguing in favor of the use of exploratory spatial data analysis, the article proposes the use of spatial autocorrelation indices for the study of presidential elections. The proposed scheme, while offering a glimpse of territorial political polarization in Mexico, allows for a valuation of the explanatory power of geographic space in electoral processes.

KEYWORDS | spatial concentration, socio-territorial transformations, spatial distribution.

Recibido el 3 de noviembre de 2012, aprobado el 21 de julio de 2013

E-mail: vlahdez@gmail.com

Correspondencia: Universidad Autónoma de Ciudad Juárez, Departamento de Arquitectura, Diseńo y Arte, Av. del Charro Norte 610 Norte, 32310, Ciudad Juárez, Chihuahua, México. 


\section{Introducción}

La división geográfica del electorado en México tiene varias lecturas, pero, desde el punto de vista espacial, puede verse como la culminación de un proceso de polarización política que tuvo su origen en 1988. Ese año surgió el sistema de los tres partidos principales que han sido protagonistas de la vida política en el país a lo largo de las últimas décadas (Aziz, 2007), conformando una nueva geografía del voto en México. Durante las elecciones presidenciales de 2000, los partidos dominantes (aquellos que obtuvieron entre 50\% y 59\% de la votación) tenían presencia a nivel de los estados ${ }^{1}$ : el Partido Revolucionario Institucional (PRI) en Nayarit y Sinaloa; y el Partido Acción Nacional (PAN) en Guanajuato. Hacia 2006 la situación había cambiado, porque el Distrito Federal y Tabasco fueron bastiones del Partido de la Revolución Democrática (PRD) y el PAN agregó Querétaro (Aziz, 2007).

En este trabajo se indaga la dimensión geográfica del voto presidencial durante las elecciones de 2012. El supuesto central recae en lo que se ha denominado "atrincheramiento localizado". Este término fue propuesto por Sue Wing y Walker (2010), haciendo referencia a los trabajos provenientes de la ciencia política, y alude al hecho de que las opiniones de los votantes tienden a operar con las preferencias ideológicas de las mayorías más cercanas. Por lo tanto, se espera que los resultados presidenciales en México muestren una tendencia al agrupamiento, reafirmando la polarización del voto de los tres principales partidos políticos.

Hoy día no son pocos los trabajos que afirman que los resultados de las elecciones no son independientes del espacio. Sobre este tema encontramos una amplia variedad de trabajos donde de forma explícita se busca explorar la hipótesis de la no aleatoriedad de los resultados electorales (Ward et al., 1996; O'Loughlin, 2002, 2003; Darmofal, 2006; Klos, 2008; Tam Cho \& Nicley, 2008; Soares \& Terron, 2008; Chen \& Rodden, 2009; Seabrook, 2009; Lefebvre \& Robin, 2009; Cutts \& Webber, 2010; Sue Wing \& Walker, 2010; Rodden, 2010; Crespin, Darmofal \& Eaves, 2011). En el contexto de México están principalmente los estudios de Vilalta (2004, 2006, 2007, 2008) y Lizama (2012).

En el campo de la geografía electoral, los trabajos se estructuran desde cinco áreas de estudio: i) la definición de las unidades espaciales (secciones, distritos, circunscripciones electorales), fase que corresponde a la organización espacial de las elecciones; ii) el análisis de las variaciones espaciales del voto, donde también se incluyen los trabajos bivariados o multivariados; iii) el examen de la influencia de los factores ambientales y espaciales en la decisión del voto; iv) el estudio de las estructuras espaciales de representación, traducida en la conformación de los puestos legislativos; y v) los estudios referentes al reparto del poder y las políticas puestas en marcha que reflejen las pautas de representación popular.

A pesar de la irrupción de la geografía electoral en la década de los sesenta del siglo $\mathrm{Xx}$, en buena medida el espacio geográfico está ausente en la mayoría de los estudios electorales, u ocupa un lugar secundario, porque solo se lo toma en cuenta

1 Para organizar, gobernar y administrar su territorio, los Estados Unidos Mexicanos se organizan en 31 estados y el Distrito Federal. 
por su valor descriptivo como unidad de análisis. Y a pesar de las evidencias empíricas, es en recientes fechas cuando la puesta en práctica de métodos que valoran la autocorrelación espacial y los enfoques de la geografía electoral, ha reubicado el espacio como una categoría explicativa.

Dos enfoques sustentan el desarrollo de este trabajo: por una parte, desde la perspectiva de la geografía electoral se busca establecer la influencia que tiene el espacio geográfico en las decisiones del voto; por otra, se emplea el análisis exploratorio de datos espaciales para determinar el valor de la correlación espacial de los resultados de la votación para Presidente de la República de México en 2012.

La valoración de la dependencia espacial ha sido aplicada a la determinación geoestadística de la correlación entre variables demográficas y comportamiento de los votantes (Klos, 2008). Vilalta $(2004,2007,2008)$ califica de indispensable, para los estudios electorales, conocer cómo se distribuye geográficamente el voto. Además, ofrece evidencias de la relevancia del espacio geográfico como variable explicativa del mismo.

Las preguntas que este trabajo busca responder son: ¿existe un patrón espacialmente aleatorio en la distribución de las elecciones presidenciales de 2012 en México?; y, por otra parte, los distritos electorales con mayor o menor votación por los candidatos, ¿están agrupados o dispersos geográficamente en el país?

El sistema de partidos políticos que participaron en el proceso electoral de 2012 en México fueron siete: Partido Acción Nacional (PAN), Partido Revolucionario Institucional (PRI), Partido de la Revolución Democrática (PRD), Partido del Trabajo (PT), Partido Verde Ecologista de México (pvem), Movimiento Ciudadano (MC) y Nueva Alianza (NA). El Consejo General del Instituto Federal Electoral (IFE) otorgó al PRI, al PVEM y a NA el registro de la coalición parcial denominada "Compromiso por México", y a los partidos PRD, PT y MC, el registro de la coalición total denominada "Movimiento Progresista". En el caso de la coalición parcial, el partido NA decidió separarse y participar de manera individual en el proceso electoral federal. Entre el 15 y 22 de marzo de 2011 se registraron cuatro candidatos para Presidente de los Estados Unidos Mexicanos (Instituto Federal Electoral [IfE], 2011b).

Luego de esta introducción, en la siguiente sección se hace una revisión de los estudios previos sobre geografía electoral, polarización electoral y autocorrelación espacial. A continuación se presenta el método de estudio, las hipótesis y las fuentes de información. En tercer lugar, los resultados del análisis espacial, concluyendo el trabajo y citando las referencias pertinentes.

\section{Geografía electoral}

La geografía electoral es una rama de la geografía política que durante los últimos cincuenta ańos fue subordinada al enfoque del comportamiento electoral. En la actualidad, la geografía electoral goza de un campo propio del conocimiento geográfico, aunque algunos autores la catalogan en el campo de la ciencia política (Sobrino, 1998). Para otros, la geografía electoral solo puede darse en dos niveles: la distribución territorial de los resultados; y el establecimiento de relaciones entre los resultados o realidad electoral y aspectos sociales, lo cultural, el nivel de desarrollo, 
incluyendo el clima (Reynoso, 1991). Destaca la definición de autores para los que una de las funciones centrales de la geografía electoral es el reconocimiento de los patrones espaciales, y el hecho de que, además de superar los aspectos meramente descriptivos, esta rama de la geografía política apunta hacia la explicación (González, 1999; Monzón, 2009).

El enfoque con mayor aceptación y desarrollo en el campo de los estudios electorales es el ecológico, que combina aspectos y técnicas cuantitativas y cualitativas con el propósito de explicar la inclinación de los votantes por una opción partidista (Bosque, 1988). Si bien este enfoque avanzó en la determinación de las preferencias a nivel de agregados espaciales (región, municipio o distrito electoral), aún quedaba pendiente el análisis del comportamiento individual. El mismo Bosque clasifica como factores individuales las preferencias de los electores por una u otra opción política. A partir de aquí, el enfoque ecológico se enriquece con la incorporación de la corriente comportamental o behaviorista (Monzón, 2009)

\section{CUADRO 1 Enfoques de geografía electoral}

\begin{tabular}{|l|l|}
\hline \multicolumn{1}{|c|}{ TEORÍA SOCIAL } & \multicolumn{1}{c|}{ GEOGRAFía } \\
\hline Geografía del comportamiento electoral. & $\begin{array}{l}\text { La organización espacial de las elecciones, con } \\
\text { especial referencia a la definición de las circuns- } \\
\text { cripciones. }\end{array}$ \\
\hline $\begin{array}{l}\text { Efecto geográfico de los flujos de información en } \\
\text { el comportamiento electoral. }\end{array}$ & $\begin{array}{l}\text { Las variaciones espaciales en las pautas del voto, } \\
\text { más las relaciones entre estas y otras características } \\
\text { poblacionales. }\end{array}$ \\
\hline $\begin{array}{l}\text { El estudio de los mecanismos y variables para la } \\
\text { división del territorio en zonas electorales. }\end{array}$ & $\begin{array}{l}\text { La influencia de los factores ambientales y espacia- } \\
\text { les en las decisiones de los votos. }\end{array}$ \\
\hline $\begin{array}{l}\text { Relaciones entre los resultados electorales y la } \\
\text { organización, plataforma y movilización de los } \\
\text { partidos políticos. }\end{array}$ & $\begin{array}{l}\text { Las estructuras espaciales de representación } \\
\text { producidas al traducirse los votos en escańos de un } \\
\text { parlamento u organismo similar. }\end{array}$ \\
\hline & $\begin{array}{l}\text { Las variaciones en el espacio del reparto de poder y } \\
\text { de la implementación de las políticas que reflejan } \\
\text { las pautas de representación. }\end{array}$ \\
\cline { 2 - 3 }
\end{tabular}

FUENTE ELABORACIÓN PROPIA CON BASE EN JOHNSTON, DEREK Y SMITH (I987); SOBRINO (I998).

Sobrino (1998) mencionaba, desde el enfoque de la teoría crítica, que el comportamiento de los resultados locales no reflejaban las categorías nacionales. Además de que los estudios de geografía electoral son importantes para constituir asociaciones estadísticas, se refería al comportamiento electoral de los individuos explicado por los fenómenos políticos. Pero en su análisis no hace referencia al espacio geográfico como una variable capaz de explicar las diferencias entre resultados locales y nacionales a las que se refiere.

Mientras para el enfoque derivado de la teoría social las unidades de análisis son diversas, abarcando desde el individuo hasta grupos sociales más amplios (Sobrino, 1998), para el enfoque geográfico las unidades de análisis se diferencian por su escala, teniéndose estudios seccionales (unidad básica de información electoral), distritales, municipales, estatales, por circunscripción o nacionales. 


\section{Polarización}

La polarización política es la separación del electorado a lo largo de una línea ideológica y/o de sus opiniones, que se muestra en la concentración de los votantes en los extremos, polos definidos con base en la posición o preferencia moderada o "central” (Sue Wing, 2009). Sin embargo, esta idea no puede ser aplicada de forma automática en México, entre otras causas, por la ausencia de una verdadera competencia electoral interpartidista. Lo que se apreció durante décadas fue una competencia entre las distintas facciones que componía el PRI (Martínez, 1997). Sin embargo, las elecciones de 2006, entre otros aspectos, recalcaron las diferencias ideológicas y la polarización como fenómenos emergentes (Magar \& Romero, 2007; Bruhn \& Greene, 2007; Moreno, 2008; Beltrán, 2009).

Magar y Romero (2007), haciendo referencia a los resultados de las elecciones presidenciales de 2006, mencionan que no es muy evidente un patrón geográfico del voto presidencial y que lo que se observa es un mosaico multipartidista, donde el voto por el PAN predomina en el norte, y por el PRD en el sur del país. Existen coincidencias entre estos autores y Martínez (1997), en cuanto a que la división del electorado en México no obedece a la tradicional separación ideológica entre izquierda y derecha. No obstante, si bien no existe consenso en México respecto de una división ideológica tradicional, tanto los candidatos como los electores han manifestado su ubicación en el eje izquierda-derecha. Los trabajos de Bruhn y Greene (2007) y de Moreno (2008) ahondaron en los resultados después de las elecciones presidenciales de 2006 y encontraron que los candidatos del PRD al Congreso se ubican en la sección izquierda del espectro ideológico; en contraste, los candidatos del pAN tienden a la sección ideológica derecha. En lo que respecta a los votantes, estos están menos polarizados que los candidatos; Bruhn y Greene (2007) mencionan que cerca del $27,5 \%$ de los votantes no se coloca en ninguna posición ideológica. Por su parte, Moreno (2008) menciona que la opinión pública, después de 2006, mostró una división de opiniones en temas de relevancia en el contexto poselectoral coincidente con la parcelación de las elites políticas.

Desde la perspectiva espacial, es posible determinar geográficamente la polarización de los votantes o el agrupamiento de las preferencias ideológicas. Es así como en diversos estudios se encuentran referencias a distritos electorales que conforman agrupamientos similares.

La Figura 1 traza las preferencias del electorado: del lado izquierdo, la tendencia liberal; y del lado derecho, la opción conservadora. En la gráfica de la izquierda la curva con forma de campana no está polarizada y muestra la mayoría de las preferencias en el centro. En contraste, la forma de la curva punteada es una distribución bimodal con la misma proporción de facciones opuestas. En cuanto a la distribución de la curva en tono gris claro, indica que los votantes se desplazan desde la opción central hacia los extremos. Lo anterior se manifiesta como una cualidad anisotrópica, en alusión a una característica física que presentan los medios cristalinos, donde la propiedad depende de la dirección del medio; es decir, la curva se desplaza según la tendencia del electorado. 
Figura 1 | Polarización electoral

Fracción del electorado

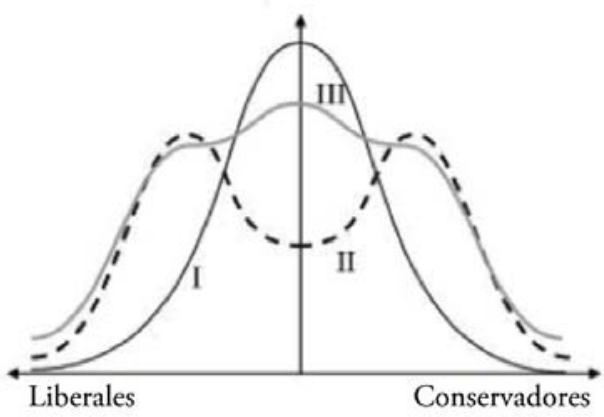

A. Anisotrópico
Fracción del electorado

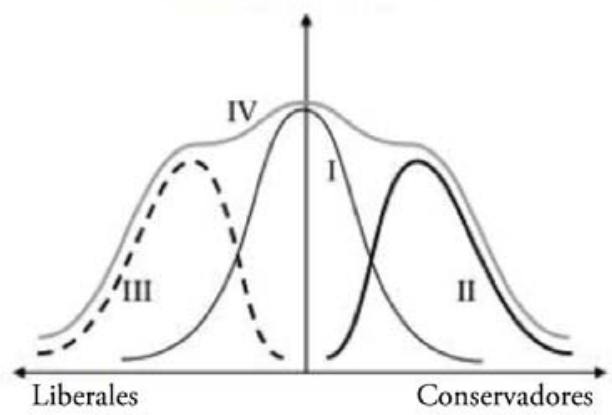

B. Aglomeración espacial

FUENTE ADAPTADO DE SUE WING (2009).

Ahora bien, la manifestación estadística de la polarización que se muestra en la sección derecha (aglomeración espacial) de la Figura 1, es un ejemplo hipotético donde los votantes son divididos en dos regiones separadas. La curva I muestra la preferencia central de los votantes, que se distribuyen en ambas regiones: campana normal. Los votantes con tendencia conservadora residen en la región de la curva II; en contraste, los votantes de tendencia liberal se encuentran en la región de la curva punteada. Se puede observar que las preferencias agregadas en la distribución de la curva de tono gris claro de la sección derecha son similares al estado intermedio de la curva gris claro de la gráfica de la izquierda, pero con un electorado que está geográficamente polarizado.

La realidad del escenario de las votaciones presidenciales en México es mucho más compleja que la explicación anterior, pero el principio general de la autocorrelación espacial y la polarización del voto subyace como fuentes conceptuales de las elecciones presidenciales de 2012.

\section{Autocorrelación espacial}

En Griffith (2009) encontramos el significado conceptual de la autocorrelación. En primer lugar, la autocorrelación es literal, es decir, la correlación en el contexto geográfico puede ser expresada en términos de la formula del coeficiente de correlación de Pearson ( $r$ ), donde los valores vecinos del atributo de "y" sustituyen a " $\mathrm{x}$ ". El resultado es un promedio para todos los pares posibles de ubicaciones de una variable. En segundo lugar, la autocorrelación puede interpretarse como un patrón espacial, haciendo hincapié en las tendencias visibles. En ellas, la autocorrelación espacial positiva perfecta sucede cuando el atributo de una variable es constante (no tiene varianza); por lo tanto, una vez que se conoce el valor constante en un lugar, se conoce en otros lugares. En contraste, la fragmentación de los valores del atributo retrata la cualidad de aleatoriedad, cero autocorrelación espacial.

El uso conceptual de mayor aceptación para la autocorrelación es su aplicación como herramienta de diagnóstico, pues tiene importantes efectos en los modelos 
inferenciales (Anselin, 1992, 1995, 1999; Anselin \& Moreno, 2003), considerando que tiende a sesgar el valor de los coeficientes del modelo. Por esto, Griffinh (2009) alude a este concepto con la denominación de un "parámetro incómodo".

El término 'correlación' alude a información redundante. Si "x" e "y" están perfectamente correlacionadas significa que si conocemos a " $x$ " también conocemos a "y". Este nivel de conocimiento o duplicidad disminuye a medida que el coeficiente se mueve hacia cero. La autocorrelación espacial extiende el sentido de información redundante para los datos georreferenciados. De aquí la relevancia del concepto, porque el valor de un atributo en un lugar determinado se puede predecir a partir de los valores de los lugares cercanos (Griffinh, 2009). La autocorrelación espacial busca conocer qué tan agrupados o dispersos se encuentran los valores de la votación presidencial, en razón de su cercanía y significancia estadística.

\section{Método}

La información para desarrollar este trabajo procedió de dos fuentes de información pública: los resultados del cómputo distrital de la elección de Presidente de los Estados Unidos Mexicanos de 2012, disponible en el portal electrónico del IFE; y la base digital del Instituto Nacional de Estadística, Geografía e Informática (INEGI), denominada Estadísticas censales a escalas geoelectorales, Censo de Población y Vivienda 2010.

Con la información obtenida se construyó una base a escala de distrito electoral uninominal ${ }^{2}$ con el total de votación y el porcentaje por partido, incluyendo las opciones resultantes de la formación de coaliciones. La Constitución Política de los Estados Unidos Mexicanos establece la división en trescientos distritos, en donde la demarcación será el resultado de dividir el total de población del país en los distritos señalados (IFE, 2011a). Se utilizaron dos programas para analizar información espacial: GeoDa, programa libre desarrollado por el Center of Geospatial Analysis and Computation de la Universidad de Arizona ${ }^{3}$; y la versión 10 de ArcMap ${ }^{\oplus}$, desarrollado por ESRI (Enviromental Systems Research Institute).

En este apartado se definen los procesos espaciales (autocorrelación y concentración) que definen una geografía electoral de la elección presidencial de 2012. Se utiliza el enfoque denominado análisis exploratorio de datos espaciales (AEDE), cuyas técnicas buscan evidenciar las estructuras espaciales. Para Anselin (1999), el AEdE puede definirse como el conjunto de técnicas que describen y visualizan las distribuciones espaciales, identifican localizaciones atípicas o atípicos espaciales (spatial outliers), descubren esquemas de asociación espacial -agrupamientos (clústeres) - o puntos calientes (hot spots), que sugieren estructuras espaciales u otras formas de heterogeneidad espacial. Entre las técnicas de AEDE empleadas en este trabajo se encuentra el índice de Morán (autocorrelación global), e indicadores locales de asociación espacial (autocorrelación local).

2 El marco geográfico electoral de México está conformado por ocho niveles jerárquicos: País (1), Circunscripción plurinominal (5), Entidad federativa (32), Distrito uninominal (300), Municipio (2.444), Sección (65.747), Localidad (212.473), Manzana (1.948.416).

3 https://geodacenter.asu.edu/ 
La autocorrelación espacial busca conocer qué tan agrupados o dispersos se encuentran los valores de la elección presidencial, en razón de su cercanía y su significancia estadística. Los trabajos realizados en México desde el enfoque del AEDE son recientes, con el primero fechado en 2004, más otros posteriores (Vilalta, 2004, 2007, 2008; Lizama, 2012). Es Vilalta (2008, p. 572) quien menciona que la geografía electoral ofrece una gran ayuda debido a la agregación de los datos, considerando al respecto que analíticamente las preferencias electorales de los individuos en un lugar no son independientes de las de otros, sino que, por el contrario, están correlacionadas.

La autocorrelación se evalúa por medio del estadístico I de Morán, que detecta cuáles áreas circundantes tienen similares o diferentes atributos (Anselin \& Hudak, 1992). La autocorrelación espacial puede ser definida como la coincidencia de ubicación espacial de valores (Anselin, 1992, 2003). Existe una autocorrelación positiva cuando los valores altos o bajos de una variable tienden a agruparse en el espacio.

El I de Morán es definido de la siguiente forma:

$$
\begin{aligned}
I_{i} & =\frac{(x i-\bar{x})}{S_{X}^{2}} \sum w i j(X j-\bar{x}) \\
S_{X}^{2} & =\frac{\sum_{j}^{n} w i j\left(X_{i}-\bar{X}\right)^{2}}{2}
\end{aligned}
$$

Un valor positivo del estadístico I de Moran significa que altos valores están rodeados por áreas con altos valores de la variable en estudio (alto-alto) o bajos valores están rodeados por áreas de bajos valores (bajo-bajo). Un valor negativo significa que bajos valores están rodeados por altos valores (bajo-alto) o altos valores están rodeados por bajos valores (alto-bajo). Lo anterior sugiere dos clases de autocorrelación espacial positiva o clusterización (alto-alto y bajo-bajo) y dos clases de correlación espacial negativa (alto-bajo y bajo-alto) (Anselin, Syabri \& Smirnov, 2002).

\section{FIgURA 2 | Diagrama de dispersión del índice de Morán}

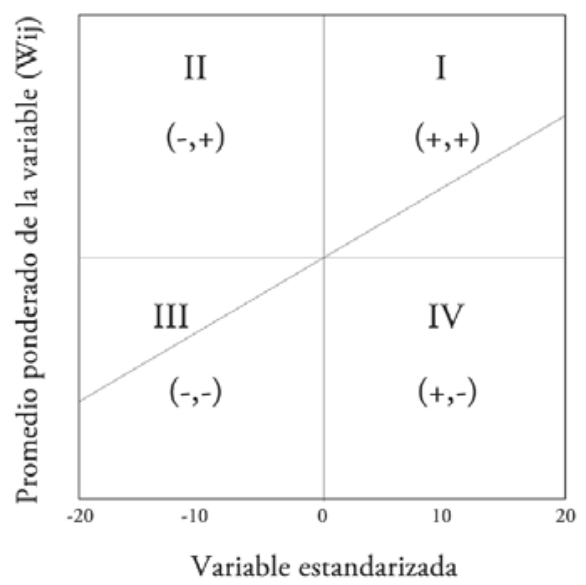

FUENTE ELABORACIÓN PROPIA CON BASE GEODA. 
El diagrama de dispersión del I de Morán (Figura 2) es la representación en un eje cartesiano de la variable analizada (voto presidencial), una vez estandarizada, y del retardo espacial de dicha variable -entiéndase el promedio ponderado de los valores que adopta una variable en el subconjunto de observaciones vecinas a una dada-. La gráfica se complementa con la representación de una recta de regresión cuya pendiente es el valor del I de Morán: mientras mayor sea la inclinación de la recta respecto del eje de las abscisas (horizontal), mayor será el valor de la autocorrelación espacial, y viceversa.

El análisis exploratorio de datos espaciales (AEDE) busca estimar la dependencia espacial entre las observaciones, para lo cual utiliza una matriz de pesos espaciales $(W)$. En la matriz de pesos, cada observación (i) se conecta con el grupo de observaciones vecinas $(j)$; por lo tanto, se obtiene una matriz del tipo Wij.

La regla de decisión para validar la hipótesis de este trabajo a partir del coeficiente de autocorrelación global (I de Morán) es:

$\mathrm{H}_{0} \mathrm{IM}=0 \quad$ El coeficiente de autocorrelación global (IM) es igual a cero, por lo que no existe un patrón espacial; se esperaría un patrón aleatorio de los votos para cada uno de los candidatos a la Presidencia de la República.

$\mathrm{H}_{1} \mathrm{IM} \neq 0 \quad$ El coeficiente de autocorrelación global (IM) es diferente a cero; por lo tanto, existe autocorrelación espacial y, en consecuencia, es posible afirmar que los resultados obtenidos por los candidatos a Presidente de la República no se distribuyen aleatoriamente en el espacio geográfico.

Sin embargo, el I de Morán es un indicador de autocorrelación global, y para visualizar y estimar estadísticamente resultados locales se requiere otro tipo de indicadores. Es decir, para estimar los agrupamientos y/o polarización del voto presidencial se propone utilizar indicadores locales de asociación espacial (ILAE - LISA, por sus siglas en inglés, correspondientes a Local Indicator of Spatial Association).

Los indicadores locales de asociación espacial son una herramienta para identificar la autocorrelación o heterogeneidad a nivel local en un área de estudio. Buscan capturar la inestabilidad de los grupos o clústeres locales en el área geográfica de estudio. La fórmula de los indicadores locales (clusters y outliers) es:

$$
I=\frac{x i-\bar{x}}{S^{2}} \sum_{j-1} w i j(x j-\bar{x})
$$

Los indicadores locales pueden tomar cualquier valor. Un valor positivo y estadísticamente significativo indica la presencia de un clúster (un distrito electoral similar a sus vecinos). A la inversa, un signo negativo sugiere que un distrito tiene valores diferentes a sus vecinos, es decir, se trata de un outlier. Los mapas ILAE (LISA) ponen de manifiesto atípicos espaciales cuya mayor o menor intensidad dependerá de la significancia asociada a los estadísticos de prueba (Getis \& Ord, 1992; Anselin, 1995; Ord \& Getis, 1995; Unwin, 2000). 


\section{Resultados y análisis}

En esta sección se valoran estadísticamente y se muestran mediante cartografías los patrones geográficos de aceptación y rechazo para cada uno de los cuatro candidatos presidenciales. La elección para designar un candidato presidencial tuvo doce opciones, debido a la naturaleza de las coaliciones. Por ejemplo, en el caso del "Movimiento Progresista": 1) prD, 2) PT, 3) MC, 4) PRD-PT-MC, 5) PRD-PT, 6) PRD-MC, 7) PT-MC; mientras que para la coalición "Compromiso por México": 8) PRI, 9) PVEM, 10) PRI-PVEM; se completan con las candidaturas individuales del: 11) PAN y 12) NA.

En el Gráfico 1 se muestran los resultados para cada uno de los siete partidos y cada una de las combinaciones resultado de las coaliciones. De forma individual, el primer lugar lo ocupa el PRI, con el 29\% del total de votos; en segunda posición está el PAN, con 26\%; y el PRD, con 19\%, ocupa la tercera posición. Los resultados finales, tomando en cuenta los votos de las coaliciones, fueron: Compromiso por México (PRI-PVem), 38,21\%; Movimiento Progresista (PRD-PT-MC), 31,59\%; el tercer candidato (PAN) obtuvo 25,41\% y el cuarto (AN), 2,29\% del total de la elección para Presidente de los Estados Unidos Mexicanos.

Cuando se revisan los resultados del promedio del voto y la desviación estándar para el conjunto de los 300 distritos electorales (véase Cuadro 2), se advierte que las diferencias entre los partidos empiezan a ser de mayor magnitud; y que entre los tres primeros porcentajes la desviación estándar del PRI es menor, es decir, la repartición del voto de este partido en el conjunto de los distritos electorales tiende a ser uniforme.

GRÁFICO 1| Porcentaje de votación presidencial, por partido y coalición, México 2012

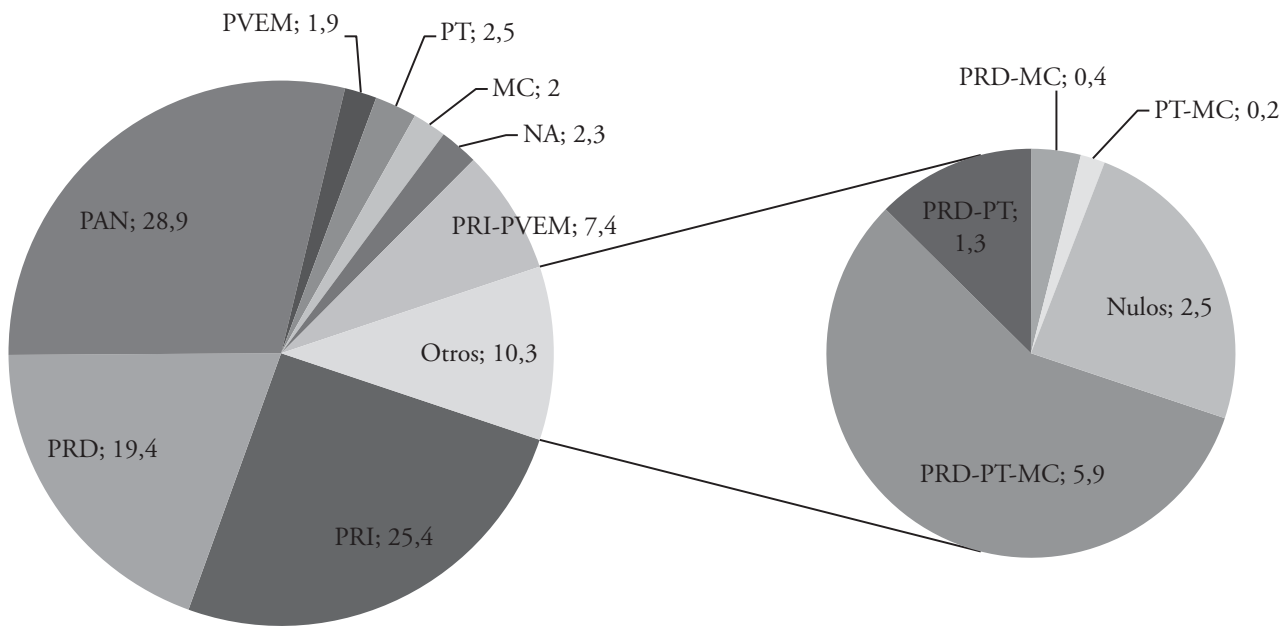

FUENTE ELABORACIÓN PROPIA CON DATOS DEL IFE 2012. 
CuAdro 2 | Elección para Presidente de México, porcentaje del voto, 2012

\begin{tabular}{|l|c|c|c|}
\hline \multicolumn{1}{|c|}{ COALICIÓN / PARTIDO } & \% VOTO & PROMEDIO & DESVIACIÓN ESTÁNDAR \\
\hline Compromiso con México & 38,21 & 38,36 & 7,57 \\
\hline Movimiento Progresista & 31,59 & 31,62 & 12,52 \\
\hline PAN & 25,41 & 25,41 & 12,78 \\
\hline Nueva Alianza & 2,29 & 2,29 & 0,99 \\
\hline
\end{tabular}

FUENTE ELABORACIÓN PROPIA CON DATOS DEL IFE 2012.

En el Cuadro 3 se observan los resultados del I de Morán ${ }^{4}$ para la elección presidencial. Estos sugieren validar la hipótesis $\left(\mathrm{H}_{1}\right)$, que considera la presencia de autocorrelación espacial en las elecciones presidenciales de 2012. Todos los coeficientes cumplen la regla de decisión que indica que $\mathrm{IM} \neq 0$ (IM diferente a cero), lo que permite rechazar la hipótesis nula $\left(\mathrm{H}_{0}\right)$. Los resultados de la elección presidencial dan cuenta de un fenómeno que posee una estructura geográfica: tienden a concentrarse en sus valores altos (cuadrante I) y en sus valores bajos (cuadrante III).

CUADRo 3 | Autocorrelación espacial de las elecciones presidenciales, México, 2012

\begin{tabular}{|l|l|c|c|}
\hline \multicolumn{1}{|c|}{ COALICIÓN / PARTIDO } & I DE MORÁN & $\begin{array}{c}\text { VALOR } \\
\text { ESPERADO }\end{array}$ & $\begin{array}{c}\text { DESVIACIÓN } \\
\text { ESTÁNDAR }\end{array}$ \\
\hline Compromiso con México (PRI-PVEM) & $0,6806^{*}$ & $-0,0033$ & 0,0398 \\
\hline Movimiento Progresista (PRD-PT-MC) & $0,8306^{*}$ & $-0,0333$ & 0,0391 \\
\hline PAN & $0,7252^{*}$ & $-0,0033$ & 0,0396 \\
\hline Nueva Alianza & $0,5056^{*}$ & $-0,0033$ & 0,0382 \\
\hline PRI & $0,6665^{*}$ & $-0,0033$ & 0,0382 \\
\hline PRD & $0,8386^{*}$ & $-0,0033$ & 0,0405 \\
\hline
\end{tabular}

*PSEUDO-VALOR O.000 I

FUENTE ELABORACIÓN PROPIA CON DATOS DEL IFE 20 I 2.

En la Figura 3 se muestra la distribución de los resultados presidenciales por distritos electorales según la relación espacial que los congrega. Estos distritos pueden agruparse en clústeres electorales, cuadrantes I y III (distritos vecinos con valores similares de votación por cada uno de los candidatos presidenciales) o en outliers electorales, cuadrantes II y IV (distritos vecinos con valores disímiles del porcentaje de votación).

El voto a favor del PRI se distribuye de manera uniforme; incluso, algunos distritos tienen la característica de votación favorable (alto-bajo: véase Figura 3). El voto del PRD y del PAN tiende a concentrarse en regiones particulares; destaca el agrupamiento del PRD en el sur del país; mientras que por el PAN se inclinan las regiones noreste, centro-occidente y golfo de México. Por lo que respecta al voto

4 La comprobación de la hipótesis se realizó con un nivel de significancia del 95\%; por lo tanto, se espera un p-valor $<0,05$ 
de Nueva Alianza (NA), su distribución es la de menor valor de autocorrelación (Cuadro 2). Destacan distritos con alto porcentaje del voto a favor de este partido desde la parte central del país hasta los distritos que pertenecen a las entidades de Chihuahua y Baja California (norte de México).

\section{FIgura 3 | Distribución espacial del I de Morán, voto presidencial,} México, 2012
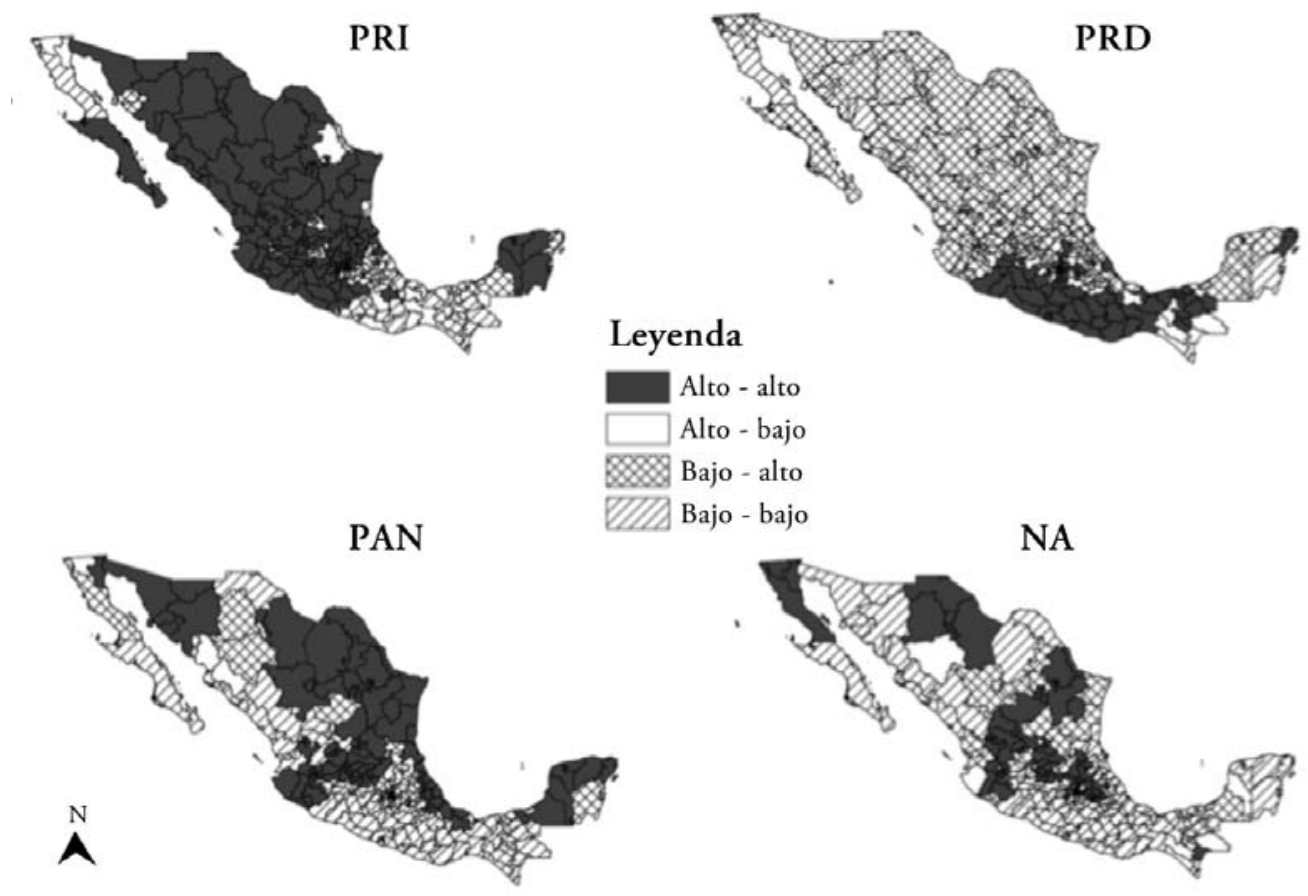

FUENTE ELABORACIÓN PROPIA CON DATOS DEL IFE 2012.

En el Cuadro 4 se destaca que los mayores porcentajes de votos se concentran en los clústeres de autocorrelación espacial (alto-alto, bajo-bajo). Mientras el PAN consiguió el mayor porcentaje acumulado entre los distritos vecinos con la característica de un alto porcentaje de votos a favor, el PRD obtuvo la opción inversa, es decir, el mayor porcentaje acumulado entre los distritos de bajo porcentaje de votación. Cabe mencionar que estos resultados no suman el 100\%, porque se está representando el porcentaje de los distritos agrupados según los gráficos de dispersión I de Morán (cuatro cuadrantes) para cada opción política.

A continuación se analizan los indicadores locales de asociación espacial (LISA) para identificar y localizar geográficamente los distritos electorales que tienden a agruparse en clústeres espaciales. El I de Morán nos plantea la interrogante de si a nivel local es posible identificar y localizar la formación de agrupamientos. Para ello se aplica el índice local de asociación espacial, que descompone localmente el índice global. Para realizar el análisis se hace referencia a la división política administrativa 
conformada por los 31 estados y el Distrito Federal. Cuando se menciona el norte del país, se hace referencia a Baja California, Baja California Sur, Sonora, Chihuahua, Coahuila, Tamaulipas, Nuevo León, Durango y Sinaloa. El suroeste está conformado por los estados de Guerrero, Oaxaca, Chiapas. El occidente incluye los estados de Colima, Nayarit, Michoacán y Jalisco. El oriente comprende Hidalgo, Puebla, Tlaxcala y Veracruz. El sureste es la región conformada por Campeche, Quintana Roo, Tabasco y Yucatán. La región central se divide en centro-norte, con Aguascalientes, Guanajuato Querétaro, San Luis Potosí y Zacatecas; y en centro-sur, que incluye el estado de México, Morelos y el Distrito Federal.

CUADro 4 Porcentaje de votación total por clúster y partido, elecciones presidenciales, México, 2012

\begin{tabular}{|l|l|l|l|c|}
\hline \multicolumn{1}{|c|}{ CLÚSTER } & PRI & PRD & PAN & NA \\
\hline Alto-alto & 7,22 & 5,54 & 8,26 & 0,32 \\
\hline Bajo-bajo & 2,65 & 2,9 & 2,62 & 0,19 \\
\hline Bajo-alto & 0,01 & 0 & 0,12 & 0,05 \\
\hline Alto-bajo & 0,01 & 0 & 0 & 0,01 \\
\hline Total & $\mathbf{9 , 8 9}$ & $\mathbf{8 , 4 4}$ & $\mathbf{1 1 , 0 0}$ & $\mathbf{0 , 5 6}$ \\
\hline
\end{tabular}

FUENTE ELABORACIÓN PROPIA CON DATOS DEL IFE 2012.

En la Figura 4 se observa, por una parte, una geografía del voto marcada por la concentración de los distritos formando clústeres espaciales de altos (tonos más oscuros) y bajos porcentajes (tonos grises claros) del voto; y por otra, los outliers (achurados).

En primer lugar, el voto para el PRI forma dos clústeres, o distritos de alto porcentaje (tono oscuro). El primero, que se extiende desde Chihuahua hasta Jalisco, incluye los estados de Sinaloa, Durango, Nayarit y Zacatecas. El segundo clúster forma un arco desde la parte occidental del estado de México, que se extiende en dirección norponiente, hasta el distrito electoral con cabecera en Tantoyuca, Veracruz. Los distritos electorales con altos porcentajes de votación a favor del PRI se localizan en 16 entidades y 56 distritos electorales, que sumaron para este partido 3.630.978 votos para Presidente de la República, el 24,93\% de su votación total (sin alianza) y 7,22\% del total de votos (Cuadro 5).

Los distritos con bajos porcentajes de votación para el PRI, y estadísticamente significativos (gris claro), se localizan en seis entidades (Cuadro 6). Destaca el clúster de 25 distritos electorales del Distrito Federal. Para el PRI, este clúster (gris claro) representó el 2,65\% de la votación total. Estos resultados dan cuenta de que el PRI tiene poca presencia en las tres principales zonas urbanas del país (en la Ciudad de México, Guadalajara y Monterrey); en cambio, el voto que lo impulsó a obtener el triunfo se fincó en zonas de menor peso demográfico (ciudades medias entre 10.000 y 100.000 habitantes) y las zonas rurales del occidente y norte del país. Otro de los factores que pueden ayudar a explicar la concentración del voto favorable (tonos 
oscuros) recae en el nivel de marginación de las zonas; en general son áreas con mediana o baja marginación ${ }^{5}$.

CUADRO 5 Porcentaje de votación presidencial por el PRI (clúster alto - alto)

\begin{tabular}{|l|c|c|c|c|c|}
\hline \multicolumn{1}{|c|}{ ENTIDAD } & $\begin{array}{c}\text { NO. } \\
\text { DISTRITO }\end{array}$ & VOTOS & \% CLÚSTER & \% PRI & $\begin{array}{c}\text { \% } \\
\text { NACIONAL }\end{array}$ \\
\hline Coahuila & 4 & 222.617 & 6,13 & 1,53 & 0,44 \\
\hline Chihuahua & 6 & 389.708 & 10,73 & 2,68 & 0,77 \\
\hline Durango & 4 & 256.067 & 7,05 & 1,76 & 0,51 \\
\hline Guanajuato & 2 & 104.476 & 2,88 & 0,72 & 0,21 \\
\hline Guerrero & 1 & 51.952 & 1,43 & 0,36 & 0,1 \\
\hline Hidalgo & 4 & 252.133 & 6,94 & 1,73 & 0,5 \\
\hline Jalisco & 7 & 432.433 & 11,91 & 2,97 & 0,86 \\
\hline Estado de México & 10 & 729.384 & 20,09 & 5,01 & 1,45 \\
\hline Michoacán & 2 & 86.380 & 2,38 & 0,59 & 0,17 \\
\hline Nayarit & 3 & 179.277 & 4,94 & 1,23 & 0,36 \\
\hline Querétaro & 1 & 66.297 & 1,83 & 0,46 & 0,13 \\
\hline San Luis Potosí & 2 & 101.697 & 2,8 & 0,7 & 0,2 \\
\hline Sinaloa & 7 & 396.046 & 10,91 & 2,72 & 0,79 \\
\hline Sonora & 1 & 49.330 & 1,36 & 0,34 & 0,1 \\
\hline Veracruz & 1 & 62.686 & 1,73 & 0,43 & 0,12 \\
\hline Zacatecas & 46 & 3.630 .978 & 100,00 & 24,93 & 0,5 \\
\hline Total & 250.495 & 6,9 & 1,72 & 7,22 \\
\hline
\end{tabular}

FUENTE ELABORACIÓN PROPIA CON DATOS DEL IFE $20 \mathrm{O} 2$.

CUADRo 6 | Porcentaje de votación presidencial por el PRI (clúster bajo-bajo)

\begin{tabular}{|l|c|c|c|c|c|}
\hline \multicolumn{1}{|c|}{ ENTIDAD } & $\begin{array}{c}\text { NO. } \\
\text { DISTRITO }\end{array}$ & VOTOS & \% CLÚSTER & \% PRI & $\begin{array}{c}\text { \% } \\
\text { NACIONAL }\end{array}$ \\
\hline Chiapas & 4 & 156.930 & 11,77 & 1,08 & 0,31 \\
\hline Distrito Federal & 25 & 827.580 & 62,1 & 5,68 & 1,64 \\
\hline Nuevo León & 1 & 33.845 & 2,54 & 0,23 & 0,07 \\
\hline Puebla & 7 & 221.734 & 16,64 & 1,52 & 0,44 \\
\hline Tlaxcala & 1 & 44.578 & 3,34 & 0,31 & 0,09 \\
\hline Veracruz & 1 & 48.084 & 3,61 & 0,33 & 0,1 \\
\hline Total & 39 & 1.332 .751 & 100,00 & 9,15 & 2,65 \\
\hline
\end{tabular}

FUENTE ELABORACIÓN PROPIA CON DATOS DEL IFE 20 I 2.

5 El Consejo Nacional de Población (Conapo) elabora el índice de marginación. Este es una medida resumen que permite diferenciar el impacto global de las carencias que padece la población como resultado de la falta de acceso a la educación, a los servicios de salud, la residencia en viviendas inadecuadas y la carencia de bienes. 
En la Figura 4 se observa el agrupamiento del voto a favor del PRD. Los valores altos-altos (tonos oscuros) forman dos clústeres. El primero es un agrupamiento que parte desde el Distrito Federal y los distritos electorales orientales del estado de México, que se extiende hacia el sur hasta los estados de Morelos, Guerrero y Oaxaca. El segundo agrupamiento coincide con el estado de Tabasco. El PRD consiguió 9.749.824 de votos para Presidente de la República. La zona "caliente" del PRD representó $28,61 \%$ y $5,54 \%$ de la votación total (Cuadro 7).

CUADRO 7 | Porcentaje de votación presidencial por el PRD (clúster alto-alto)

\begin{tabular}{|l|r|r|r|r|c|}
\hline \multicolumn{1}{|c|}{ ENTIDAD } & $\begin{array}{c}\text { NO. } \\
\text { DISTRITO }\end{array}$ & VOTOS & \% CLÚSTER & \% PRI & $\begin{array}{c}\text { \% } \\
\text { NACIONAL }\end{array}$ \\
\hline Distrito Federal & 23 & 1.276 .571 & 45,76 & 13,09 & 2,54 \\
\hline Guerrero & 8 & 396.763 & 14,22 & 4,07 & 0,79 \\
\hline Estado de México & 5 & 215.371 & 7,72 & 2,21 & 0,43 \\
\hline Morelos & 3 & 136.211 & 4,88 & 1,4 & 0,27 \\
\hline Oaxaca & 7 & 295.946 & 10,61 & 3,04 & 0,59 \\
\hline Tabasco & 6 & 468.904 & 16,81 & 4,81 & 0,93 \\
\hline Total & 52 & 2.789 .766 & 100,00 & 28,61 & 5,54 \\
\hline
\end{tabular}

FUENTE ELABORACIÓN PROPIA CON DATOS DEL IFE $20 \mathrm{I} 2$.

FIGURA 4 Clústeres del porcentaje de votación en las elecciones presidenciales, México, 2012
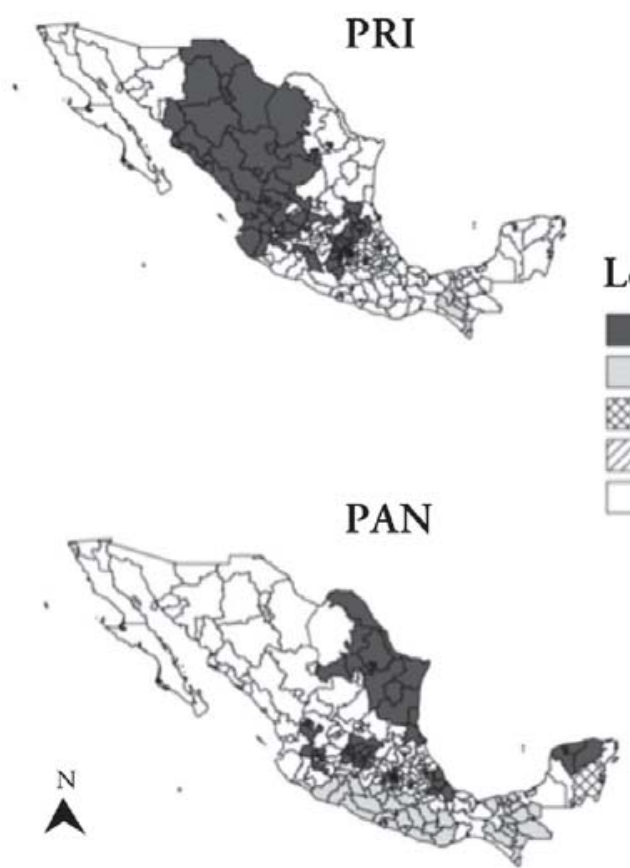

Leyenda

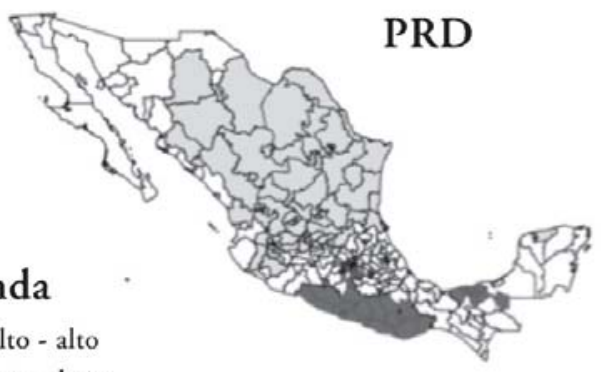

Bajo - bajo

Bajo - alto

QIIA Alto - bajo

Sin significancia

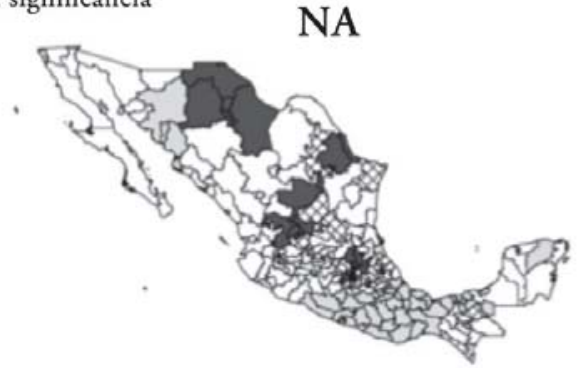


La zona "fría" del PRD (bajo-bajo) se extiende por 16 entidades del país, que suman 76 distritos electorales. En esta zona, el PRD agenció 14,99\% de su votación total, que representó el 2,9\% de la votación total (Cuadro 7). Al observar la distribución espacial del voto a favor del PRD, destacan tres factores.

El primero corresponde al bastión de la Ciudad de México -sede de los poderes Ejecutivo, Legislativo y Judicial de México, además de ser la mayor concentración urbana del país y con el más bajo nivel de marginación-, que desde las elecciones de 1988 ha sido la palestra donde los candidatos del PRD tienen en común, primero, ser jefes de gobierno de la Ciudad de México, y después, candidatos a la presidencia.

Un segundo factor destacable es que, a partir de la Ciudad de México, pero con dirección sur, el segundo agrupamiento del PRD marca un contraste con la Ciudad de México, porque es una zona donde predominan altos y muy altos niveles de marginación, zonas rurales y de difícil acceso, debido a su topografía (montañas).

Y en tercer lugar, destaca el distrito del estado de Tabasco, donde el voto a favor se explica por tratarse de la región de nacimiento del candidato por el PRD, Andrés Manuel López Obrador; es decir, existe un marcado regionalismo del voto en los dos casos analizados hasta el momento, PRI y PRD.

CUADRO 8 | Porcentaje de votación presidencial por el PRD (clúster bajo-bajo)

\begin{tabular}{|l|c|c|c|c|c|}
\hline \multicolumn{1}{|c|}{ ENTIDAD } & $\begin{array}{c}\text { NO. } \\
\text { DISTRITO }\end{array}$ & VOTOS & \% CLÚSTER & \% PRI & \% NACIONAL \\
\hline Aguascalientes & 3 & 60.681 & 4,15 & 0,62 & 0,12 \\
\hline Coahuila & 6 & 113.735 & 7,78 & 1,17 & 0,23 \\
\hline Chihuahua & 4 & 74.247 & 5,08 & 0,76 & 0,15 \\
\hline Durango & 4 & 64.483 & 4,41 & 0,66 & 0,13 \\
\hline Guanajuato & 13 & 189.179 & 12,94 & 1,94 & 0,38 \\
\hline Jalisco & 18 & 301.835 & 20,65 & 3,1 & 0,6 \\
\hline Michoacán & 1 & 15.041 & 1,03 & 0,15 & 0,03 \\
\hline Nayarit & 1 & 25.798 & 1,77 & 0,26 & 0,05 \\
\hline Nuevo León & 9 & 181.292 & 12,4 & 1,86 & 0,36 \\
\hline Querétaro & 2 & 62.549 & 4,28 & 0,64 & 0,12 \\
\hline San Luis Potosí & 5 & 93.992 & 6,43 & 0,96 & 0,19 \\
\hline Sinaloa & 2 & 45.951 & 3,14 & 0,47 & 0,09 \\
\hline Tamaulipas & 5 & 107.526 & 7,36 & 1,1 & 0,21 \\
\hline Veracruz & 1 & 15.763 & 1,08 & 0,16 & 0,03 \\
\hline Yucatán & 1 & 20.749 & 1,42 & 0,21 & 0,04 \\
\hline Zacatecas & 4 & 88.680 & 6,07 & 0,91 & 0,18 \\
\hline Total & 79 & 1.461 .501 & 100,00 & 14,99 & 2,9 \\
\hline
\end{tabular}

FUENTE ELABORACIÓN PROPIA CON DATOS DEL IFE $20 \mathrm{I} 2$.

El voto para el PAN, con valores altos-altos, se caracteriza por agruparse en cinco clústeres: el de mayor extensión se localiza en el noroeste del país y se extiende por el golfo de México; el segundo se ubica en los distritos centrales de Veracruz; 
a continuación está el agrupamiento que se forma con los distritos electorales de Yucatán; el cuarto clúster comprende las entidades de Querétaro y Guanajuato; finalmente está el agrupamiento en distritos del estado de Jalisco, en el centrooccidente del país (Figura 4).

CUADRo 9 | Porcentaje de votación presidencial por el PAN (clúster alto-alto)

\begin{tabular}{|l|c|c|c|c|c|}
\hline \multicolumn{1}{|c|}{ ENTIDAD } & $\begin{array}{c}\text { NO. } \\
\text { DISTRITO }\end{array}$ & VOTOS & \% CLÚSTER & \% PRI & $\begin{array}{c}\text { \% } \\
\text { NACIONAL }\end{array}$ \\
\hline Coahuila & 3 & 187.447 & 4,51 & 1,47 & 0,37 \\
\hline Guanajuato & 14 & 950.197 & 22,86 & 7,43 & 1,89 \\
\hline Jalisco & 8 & 471.675 & 11,35 & 3,69 & 0,94 \\
\hline Nuevo León & 11 & 766.711 & 18,45 & 6 & 1,52 \\
\hline Querétaro & 2 & 163.304 & 3,93 & 1,28 & 0,32 \\
\hline Tamaulipas & 8 & 600.731 & 14,45 & 4,7 & 1,19 \\
\hline Veracruz & 9 & 620.422 & 14,93 & 4,85 & 1,23 \\
\hline Yucatán & 5 & 395.678 & 9,52 & 3,09 & 0,79 \\
\hline Total & $\mathbf{6 0}$ & $\mathbf{4 . 1 5 6 . 1 6 5}$ & $\mathbf{1 0 0}$ & $\mathbf{3 2 , 5}$ & $\mathbf{8 , 2 6}$ \\
\hline
\end{tabular}

FUENTE ELABORACIÓN PROPIA CON DATOS DEL IFE $20 \mathrm{I} 2$.

El PAN consiguió 12.786.647 votos para Presidente de la República. De estos, el $32,50 \%$ lo obtuvo en los clústeres altos-altos; comparados con el porcentaje de votación total (nacional), su valor fue de $8,26 \%$ (Cuadro 9). En cuanto al número de distritos "calientes" o "fríos", para el PAN no hay diferencia: 60 en el primer caso y 61 en el segundo (Cuadros 9 y 10). Pero la brecha en votos se manifiesta en el porcentaje total de votación nacional alcanzado en cada clúster: como se mencionó, 8,26\% en el clúster alto-alto, en contra de 2,62\% en el clúster bajo-bajo. Mientras que el PRI carece de una presencia importante en las tres principales zonas urbanas de México (ciudad de México, Guadalajara y Monterrey), el PAN tiene un importante porcentaje de sus votos en las ciudades y zonas de influencia de Guadalajara (centro-occidente del país) y Monterrey (noreste), ambas regiones con bajos y muy bajos niveles de marginación y sedes de importantes grupos empresariales. Al igual que en los casos previamente analizados, para el PAN también se observa una región tradicional del voto, "la región del Bajío mexicano", que comprende la región circunvecina al estado de Guanajuato (cabe mencionar, región de origen del expresidente Vicente Fox, presidente de México durante el sexenio 2000-2006, candidato por el PAN).

En lo que respecta a los porcentajes altos-altos de la votación por Nueva Alianza, los valores con significancia se agrupan en cuatro bloques (Figura 4). El primer agrupamiento, en ocho distritos de Chihuahua (norte); el segundo en el noroeste, en los límites de Coahuila y Tamaulipas; el tercero en la parte central de México, en las entidades de Zacatecas y Aguascalientes; finalmente, cinco distritos de Hidalgo y uno del estado de México conforman el agrupamiento restante.

El partido Nueva Alianza alcanzó la cifra de 1.150.662 votos para Presidente de la República. En el Cuadro 10 se observa que los votos de esta organización política representaron el 13,98\% en el clúster alto-alto, y solo $0,32 \%$ de la votación 
total. En el estado de Chihuahua se concentró el mayor porcentaje de votación $(3,86 \%)$. En el Cuadro 11 se muestran los resultados para el clúster bajo-bajo. En primera instancia, se observa que la relación de distritos con votación "caliente" y distritos con votación "fría" es de 2 a 1 . En la entidad de Sinaloa, uno de los distritos con significancia estadística, se registraron 3.237 votos, el $0,01 \%$ de la votación total. Las zonas previamente indicadas para este partido registran mediana o baja marginación.

CUADRO 10 Porcentaje de votación presidencial por el PAN (clúster bajo-bajo)

\begin{tabular}{|l|c|c|c|c|c|}
\hline \multicolumn{1}{|c|}{ ENTIDAD } & $\begin{array}{c}\text { NO. } \\
\text { DISTRITO }\end{array}$ & VOTOS & \% CLÚSTER & \% PRI & $\begin{array}{c}\text { \% } \\
\text { NACIONAL }\end{array}$ \\
\hline Chiapas & 7 & 166.245 & 12,62 & 1,3 & 0,33 \\
\hline Chihuahua & 1 & 36.824 & 2,8 & 0,29 & 0,07 \\
\hline Distrito Federal & 8 & 170.487 & 12,94 & 1,33 & 0,34 \\
\hline Guerrero & 9 & 154.018 & 11,69 & 1,2 & 0,31 \\
\hline Estado de México & 15 & 352.842 & 26,79 & 2,76 & 0,7 \\
\hline Michoacán & 6 & 155.543 & 11,81 & 1,22 & 0,31 \\
\hline Morelos & 2 & 50.003 & 3,8 & 0,39 & 0,1 \\
\hline Oaxaca & 6 & 145.300 & 11,03 & 1,14 & 0,29 \\
\hline Puebla & 1 & 20.783 & 1,58 & 0,16 & 0,04 \\
\hline Tabasco & 6 & 65.073 & 4,94 & 0,51 & 0,13 \\
\hline Total & 61 & 1.317 .118 & 100 & 10,3 & 2,62 \\
\hline
\end{tabular}

FUENTE ELABORACIÓN PROPIA CON DATOS DEL IFE 20 I 2.

CUADRO 11| Porcentaje de votación presidencial por el NA (clúster alto-alto)

\begin{tabular}{|l|c|c|c|c|c|}
\hline \multicolumn{1}{|c|}{ ENTIDAD } & $\begin{array}{c}\text { NO. } \\
\text { DISTRITO }\end{array}$ & vOTOS & \% CLÚSTER & \% PRI & $\begin{array}{c}\text { \% } \\
\text { NACIONAL }\end{array}$ \\
\hline Aguascalientes & 3 & 33.706 & 0,07 & 2,93 & 0,07 \\
\hline Coahuila & 1 & 4.998 & 0,01 & 0,43 & 0,01 \\
\hline Chihuahua & 8 & 44.394 & 0,09 & 3,86 & 0,09 \\
\hline Guanajuato & 1 & 4.973 & 0,01 & 0,43 & 0,01 \\
\hline Hidalgo & 5 & 38.598 & 0,08 & 3,35 & 0,08 \\
\hline Estado de México & 1 & 5.932 & 0,01 & 0,52 & 0,01 \\
\hline Nuevo León & 1 & 6.940 & 0,01 & 0,6 & 0,01 \\
\hline Tamaulipas & 1 & 5.386 & 0,01 & 0,47 & 0,01 \\
\hline Zacatecas & 3 & 15.908 & 0,03 & 1,38 & 0,03 \\
\hline Total & 24 & $\mathbf{1 6 0 . 8 3 5}$ & $\mathbf{0 , 3 2}$ & $\mathbf{1 3 , 9 8}$ & $\mathbf{0 , 3 2}$ \\
\hline
\end{tabular}

FUENTE ELABORACIÓN PROPIA CON DATOS DEL IFE $20 \mathrm{I} 2$. 
CUADRo 12| Porcentaje de votación presidencial por el NA (clúster bajo-bajo)

\begin{tabular}{|l|c|c|c|c|c|}
\hline \multicolumn{1}{|c|}{ ENTIDAD } & $\begin{array}{c}\text { NO. } \\
\text { DISTRITO }\end{array}$ & VOTOS & \% CLÚSTER & \% PRI & \% NACIONAL \\
\hline Distrito Federal & 3 & 9.187 & 0,02 & 0,8 & 0,02 \\
\hline Guerrero & 9 & 20.219 & 0,04 & 1,76 & 0,04 \\
\hline Oaxaca & 10 & 14.963 & 0,03 & 1,3 & 0,03 \\
\hline Puebla & 2 & 4.989 & 0,01 & 0,43 & 0,01 \\
\hline Sinaloa & 1 & 3.237 & 0,01 & 0,28 & 0,01 \\
\hline Sonora & 3 & 5.952 & 0,01 & 0,52 & 0,01 \\
\hline Tabasco & 6 & 5.771 & 0,01 & 0,5 & 0,01 \\
\hline Veracruz & 12 & 26.547 & 0,05 & 2,31 & 0,05 \\
\hline Yucatán & 1 & 3.556 & 0,01 & 0,31 & 0,01 \\
\hline Total & 47 & $\mathbf{9 4 . 4 2 1}$ & $\mathbf{0 , 1 9}$ & $\mathbf{8 , 2 1}$ & $\mathbf{0 , 1 9}$ \\
\hline
\end{tabular}

FUENTE ELABORACIÓN PROPIA CON DATOS DEL IFE 2012.

\section{Conclusiones}

En este trabajo se evidencia la existencia de una geografía electoral del voto presidencial caracterizada por la concentración de los distritos electorales con mayor y menor porcentaje por cada uno de los candidatos presidenciales. Esto permite afirmar que los resultados de la elección presidencial no apuntan a un proceso espacial aleatorio, sino que, por el contrario, se encuentran regionalizados. Tal conclusión se inserta, por un lado, en la discusión sobre las posibilidades de la vida democrática del país, atendiendo a su pluralidad electoral; y por otra -tema central de este trabajo-, en el debate teórico y de método que busca revalorizar la relevancia del espacio geográfico en los estudios electorales.

La integración del espacio geográfico en los estudios electorales genera un cambio importante en los enfoques y metodologías que trabajan con datos electorales agrupados en unidades espaciales. Dando paso a una regionalización del voto presidencial, se puede señalar que en los distritos electorales de México, la participación electoral no se distribuye aleatoriamente en el espacio, sino que se presenta con una geografía que tiende a la concentración y autocorrelación espacial.

El espacio geográfico distrital es, así, una variable explicativa de las elecciones presidenciales de 2012. Los resultados revelan un patrón espacial de la distribución de voto y confirman la existencia de zonas tradicionales del voto para cada uno de los tres principales partidos políticos en México:

- zonas rurales y ciudades medias para el PRI, con medios y bajos niveles de marginación socioeconómica;

- para el PRD contrastan los votos de la Ciudad de México, con el más bajo nivel de marginación, y la zona rural y marginada de los estados del sur del país, principalmente Guerrero y Oaxaca; de todos los votos, los que favorecen al PRD son los más concentrados; 
- y para el pan, el voto de las dos restantes ciudades y regiones más importantes del país, las zonas metropolitanas de Guadalajara y Monterrey.

Este trabajo aporta nuevas hipótesis que ayudarían a explicar las preferencias por uno u otro partido político en función de los niveles de marginación, factores de identidad (por ejemplo, lugar de nacimiento de los candidatos a la Presidencia), grado de urbanización (tamaño de las localidades). Es decir, los estudios con el método empleado en este trabajo permiten formular nuevas hipótesis y aportar mayor conocimiento para un fenómeno multicausal como es el voto. La evidencia mostrada en este estudio confirma que las preferencias por cada uno de los candidatos y, en correspondencia, por los partidos políticos que los apoyaron, se excluyen espacialmente.

\section{Referencias bibliográficas}

Anselin, L. (1992). Spatial data analysis with GIS: An introduction to application in the social sciences. Technical Report, 92(10). Barbara, CA: National Center for Geographic Information and Analysis (NCGIA), University of California. En http://www.ncgia. ucsb.edu/Publications/Tech_Reports/92/92-10.PDF

Anselin, L. (1995). Local Indicators of Spatial Association-LISA. Geographical Analysis, 27(2), 93-115. doi: 10.1111/j.1538-4632.1995.tb00338.x

Anselin, L. (1999). Spatial Data Analysis with SpaceStat ${ }^{\mathrm{TM}}$ and ArcView. Workbook (3rd ed.). Urbana, Il: Department of Agricultural and Consumer Economics, University of Illinois. En http://161.45.251.150/s-drive/TEFF/workbook_ed3.pdf

Anselin, L. \& Hudak, S. (1992). Spatial econometrics in practice. A review of software options. Regional Science and Urban Economics, 22(3), 509-536. doi: 10.1016/01660462(92)90042-Y

Anselin, L. \& Moreno, R. (2003). Properties of tests for spatial error components. Regional Science and Urban Economics, 33(5), 595-618. doi: 10.1016/S0166-0462(03)00008-5

Anselin, L., Syabri, I. \& Smirnov, O. (2002). Visualizing multivariate spatial correlation with dynamically linked windows. Urbana, Il: Regional Economics Applications Laboratory (REAL), University o Illinois. En https://geodacenter.asu.edu/pdf/multi_lisa.pdf

Aziz, A. (2007). El retorno del conflicto. Elecciones y polarización política en México. Desacatos, mayo-agosto, 24, 13-54. En http://www.redalyc.org/articulo.oa?id=13902402

Beltrán, U. (2009). Ideología y polarización en la elección de 2006. Política y gobierno, volumen temático 2009, Elecciones en México, 83-106. En http://bit.ly/1iFY5gr

Bosque, J. (1988). Geografía electoral. Madrid: Editorial Síntesis.

Bruhn, K. \& Greene, K. (2007). Optimismo moderado: cómo podría desvanecerse el conflicto partidista mexicano. Foreign Affairs en Español, 7(1), 132-143. Versión digitalizada disponible en http://bit.ly/1qJ83QE

Chen, J. \& Rodden, J. (2009). Tobler's law, urbanization, and electoral bias: Why compact, contiguous district are bad for the democrats. Working Paper. En http://web.stanford. edu/ -jrodden/chen_rodden_florida.pdf 
Crespin, M., Darmofal, D. \& Eaves, C. (2011). The political geography of congressional elections. Annual Meeting of the Midwest Political Science Association, Chicago, IL, marzo 31-abril 3, 2011. En http://crespin.myweb.uga.edu/CrespinDarmofalEaves.pdf

Cutts, D. \& Webber, D. J. (2010). Voting patterns, Party spending and relativelocation in England and Wales. Regional Studies, 44(6), 735-760. doi: 10.1080/00343400903107744

Darmofal, D. (2006). Spatial econometrics and political science. Annual Metting of the Southern Political Science Association, Atlanta, GA, USA: The Society for Political Methodology. En http://web.cenet.org.cn/upfile/103632.pdf

Getis, A. \& Ord, J. (1992). The analysis of spatial association by use of distance statistics. Geographical Analysis, 24(3), 189-206. doi: 10.1111/j.1538-4632.1992.tb00261.x

González, J. (1999). Geografía electoral de Chile: comportamiento del electorado chileno entre 1932 / 1992. Estudios Geográficos, 60(234), 121-138.

Griffith, D. A. (2009). Spatial autocorrelation. University of Texas at Dallas, Richardson, TX, Elsevier Inc. En http://www.elsevierdirect.com/brochures/hugy/SampleContent/ Spatial-Autocorrelation.pdf

Instituto Federal Electoral (IFE). (2011a). Guía para el uso e interpretaciones de los productos cartográficos. México: Autor. En http://www.ife.org.mx/docs/IFE-v2/DERFE/DERFEDistritosElectorales/DERFE-Circunscripciones-mapas/6-GuiaCartografia.pdf

Instituto Federal Electoral (IFE). (2011b). Acuerdo del Consejo General del Instituto Federal Electoral por el que se registran las candidaturas a Presidente de los Estados Unidos, que presentan los partidos Acción Nacional y Nueva Alianza, así como las coaliciones Compromiso por México y Movimiento Progresista con el fin de participar en el proceso electoral federal 2011-2012. México: Autor. En http://www.ife.org.mx/docs/ IFE-v2/DS/DS-CG/DS-SesionesCG/CG-acuerdos/2012/Marzo/CGes201203-29/ CGes290312ap1.pdf

Instituto Federal Electoral (IFE). (2012). Sistema de Cómputos Distritales, Entidad Federativa y de Circunscripción. México: Autor. En http://computos2012.ife.org.mx/reportes/ presidente/distritalPresidenteEF.html

Instituto Nacional de Estadística, Geografía e Informática (INEGI). (2012). Estadísticas censales a escalas geoelectorales, Censo de Población y Vivienda 2012. México: Autor. En http://gaia.inegi.org.mx/geoelectoral/viewer.html\#

Johnston, R., Gregory, D. \& Smith, D. (1987). Diccionario de geografia humana (2a ed.). Madrid: Alianza Editorial.

Klos, J. (2008). Determining spatial correlations between voting behavior and selected demographic variables in a changing electorate. Middle State Geographer, 41, 19-26. En https://gis.e-education.psu.edu/sites/default/files/capstone/klos_20081201.pdf

Lefebvre, B. \& Robin, C. (2009). Pre-electoral coalitions, party system and electoral geography: A decade of general elections in India (1999-2009). South Asia Multidisciplinary Academic Journal, 3. En http://samaj.revues.org/2795

Lizama, G (2012). Geografía electoral del abstencionismo en los municipios de México (19942009). Espacialidades [Revista electrónica], 2(2), 22-51. En http://pac.ife.org.mx/ colmex2012/docs/07_Lizama.pdf

Magar, E. \& Romero, V. (2007). México: la accidentada consolidación democrática. Revista de Ciencia Politica, 27, número especial, 183-204. http://dx.doi.org/10.4067/S0718090X2007000100011 
Martínez, A. (1997). Diputados, clivajes (cleavages) y polarización en México. Perfiles Latinoamericanos, 11, 43-70. En http://www.redalyc.org/articulo.oa?id=11501103

Monzón, N (2009). Geografía electoral. Consideraciones teóricas para el caso argentino. Cuadernos de Geografia - Revista Colombiana de Geografia, 18, 119-128. En http:// www.redalyc.org/articulo.oa?id $=281822001010$

Moreno, A. (2008). La opinión pública en el contexto postelectoral de 2006. Perfiles Latinoamericanos, 16(31), 39-63.

O'Loughlin, J. (2002). The electoral geography of Weimar Germany: Exploratory Spatial Data Analysis (ESDA) of protestant support for the Nazi Party. Political Analysis, 10(3), 217-243. En http://www.colorado.edu/ibs/pec/johno/pub/nazi_pa.pdf

O’Loughlin, J. (2003). Spatial analysis in political geography. En J. Agnew, K. Mitchell \& G. Toal (Eds.), A Companion to Political Geography (pp. 30-46). Malden, MA: Blackwell Plublishing.

Ord, J. K. \& Getis, A. (1995). Local spatial autocorrelation statistics: distributional issues and an application. Geographical Analysis, 27(4), 286-306. doi: 10.1111/j.15384632.1995.tb00912.x

Reynoso, V. M. (1991). Notas para una geografía electoral del estado de Sonora. Revista de Ciencias Sociales y Humanidades, 11(22), 87-116. En http://bit.ly/1jOroIB

Rodden, J. (2010). The geographic distribution of political preferences. Annual Review of Political Science, 13, 321-340. En http://web.stanford.edu/ -jrodden/annurev. polisci.12.031607.pdf

Seabrook, N. (2009). The Obama effect: Patterns of geographic clustering in the 2004 and 2008 presidential elections. The Forum, 7(2). doi: 10.2202/1540-8884.1308

Soares, G. A. D. \& Terron, S. L. (2008). Dois Lulas: A geografia eleitoral da reeleição (explorando conceitos, métodos e técnicas de análise geoespacial). Opiniäo Pública, 4(2), 269-301. http://dx.doi.org/10.1590/S0104-62762008000200001

Sobrino, J. (1998). Elecciones municipales en el Estado de México: un análisis del contexto local. Economía, Sociedad y Territorio, 1(3), 547-595. En http://www.redalyc.org/ articulo.oa?id $=11110305$

Sue Wing, I. \& Walker J. L. (2010). The geographic dimensions of electoral polarization in the 2004 U.S. Presidential Vote. En A. Páez, J. Gallo, R. N. Builiung, S. Dall'erba (Eds.), Progress in Spatial Analysis, Methods and Applications (pp. 253-285). Berlín: Springer. Artículo disponible en http://people.bu.edu/isw/papers/the_2004_election.pdf

Tam Cho, W. K. \& Nicley, E. P. (2008). Geographic proximity versus institutions evaluating borders as real political boundaries. American Politics Research, 36(6), 803-823. doi: $10.1177 / 1532673$ X08316701

Unwin, A. (2000). Using your eyes-making statistics more visible with computers. Computational Statistics \& Data Analysis, 32, 303-312. doi: 10.1016/S0167-9473(99)00083-3

Vilalta, C. (2004). The local context and the spatial diffusion of multiparty competition in urban Mexico, 1994-2000. Political Geography, 23(4), 403-423. doi: 10.1016/j. polgeo.2003.12.009

Vilalta, C. (2006). Sobre la espacialidad de los procesos electorales urbanos y una comparación entre las técnicas de regresión OLS y SAM. Estudios Demográficos y Urbanos, 21(1), 83-122. En http://www.redalyc.org/articulo.oa?id=31200103 
Vilalta, C. (2007). El voto en una ciudad de empresarios ricos y empleados pobres: Marginación, segregación espacial y resultados electorales en la ciudad de México (1995-2000). Working Paper, EGAP 2007, Tecnológico de Monterrey. En http://alejandria.ccm. itesm.mx/egap/documentos/EGAP-2007-06.pdf

Vilalta, C. (2008). ¿'Se pueden predecir geográficamente los resultados electorales? Una aplicación del análisis de clusters y outliers espaciales. Estudios Demográficos y Urbanos, 23(3), 571-613. En http://www.redalyc.org/articulo.oa?id=31211538005

Ward, M. D., O’Loughlin, J., Shin, M., Lofdahl, C. L., Gleditsch, K. S. \& Cohen, J. S. (1996). The spatial and temporal diffusion of democracy, 1946-1994. Annual Conference of the International Studies Association, San Diego, California, USA. En http://colorado.edu/ IBS/GAD/Manuscripts/isa1996.pdf 